\section{Um modelo bayesiano para investigação de sobremortalidade durante epidemia de dengue na Região Metropolitana do Rio de Janeiro, Brasil, 2007-2008}

\author{
A Bayesian model to investigate excess \\ mortality during the dengue epidemic in Greater \\ Metropolitan Rio de Janeiro, Brazil, in 2007-2008
}

Un modelo bayesiano para la investigación del exceso de mortalidad durante la epidemia de dengue en la región metropolitana de Río de Janeiro, Brasil, 2007-2008
Thainá Alves Malhão ${ }^{1}$

Camila Maria Casquilho Resende 2

Dani Gamerman 2

Roberto de Andrade Medronho 1

\footnotetext{
${ }^{1}$ Instituto de Estudos em Saúde Coletiva, Universidade Federal do Rio de Janeiro, Rio de Janeiro, Brasil.

2 Instituto de Matemática, Universidade Federal do Rio de Janeiro, Rio de Janeiro, Brasil.

Correspondência T. A. Malhão

Instituto de Estudos em Saúde Coletiva, Universidade Federal do Rio de Janeiro.

Av. Brigadeiro Trompowski s/n, Rio de Janeiro, RJ 21949-900, Brasil.

thainaalvesmalhao@gmail. com
}

\begin{abstract}
The aim of this study was to investigate excess mortality from dengue in Greater Metropolitan Rio de Janeiro, Brazil, during an epidemic in 2007-2008. A Poisson dynamic model was tested to predict the number of deaths during these epidemic years. Inference was conducted with a Bayesian approach. Excess mortality was detected in March 2008 in children $\leq 15$ years. In addition, the highest number of reported dengue cases in Rio de Janeiro was in March and April 2008. Since the increase in mortality should be preceded by an increase in morbidity, one can hypothesize that there was excess mortality from dengue in children during the epidemic in Greater Metropolitan Rio de Janeiro in March 2008.
\end{abstract}

Dengue; Communicable Diseases; Mortality; Epidemiological Surveillance

\section{Resumo}

O objetivo deste estudo é investigar a ocorrência de sobremortalidade durante a epidemia de dengue ocorrida na Região Metropolitana do Rio de Janeiro, Brasil, entre 2007 e 2008. Para obter a predição dos óbitos desses anos epidêmicos, um modelo dinâmico Poisson foi ajustado aos dados de mortalidade por dengue e por doenças que fazem parte do seu diagnóstico diferencial. Toda a inferência foi realizada sob o paradigma bayesiano. Verificou-se um excesso de mortalidade em março de 2008, em menores de 15 anos de idade. Paralelamente, em março e abril de 2008, houve o maior número de casos notificados de dengue no Estado do Rio de Janeiro. Considerando que o aumento da mortalidade deve ser precedido por um incremento na morbidade, pode-se levantar a hipótese de que houve um excesso de mortalidade por dengue em crianças na epidemia de dengue ocorrida na Região Metropolitana do Rio de Janeiro, em março de 2008.

Dengue; Doenças Transmissíveis; Mortalidade; Vigilância Epidemiológica 


\section{Introdução}

Estudos recentes têm evidenciado uma expressiva subnotificação de casos de dengue no Sistema de Informações de Agravos de Notificação (SINAN), que representa um dos principais problemas enfrentados pela vigilância epidemiológica desse agravo $1,2,3,4,5$.

Uma investigação realizada no Município do Rio de Janeiro, com o objetivo de analisar a confiabilidade do diagnóstico final dos casos de dengue informados no SINAN durante a epidemia 2001/2002, encontrou, além dessa limitação, outros problemas como o retardo da entrada de dados no sistema de informação, o elevado percentual de informações ignoradas e de inconsistências entre os campos e a baixa qualidade da informação nos óbitos 1.

Pesquisa realizada com os dados brasileiros de mortalidade por dengue, de 2000 a 2005, corrobora essas informações ao identificar baixa concordância entre os óbitos por dengue registrados no SINAN e no Sistema de Informações sobre Mortalidade (SIM) - entre 0\% e 33\%. Esse mesmo estudo também verificou baixas coberturas para notificação de óbitos por dengue nos sistemas de informação avaliados (SINAN: entre 25,9\% e 63,4\%; SIM: entre $11,3 \%$ e $27,5 \%) 6$.

Além disso, a Organização Mundial da Saúde (OMS) e a Organização Pan-Americana da Saúde (OPAS) estimam que, durante uma epidemia de dengue, os casos notificados podem representar apenas cerca de $10 \%$ do total real de doentes 4 .

Considerando ainda a dificuldade no diagnóstico desse agravo, tanto pela sintomatologia inespecífica e similaridade do quadro clínico com outras doenças infecciosas 5,6,7 quanto pelo rigoroso critério de definição de caso utilizado pela OMS 8, pelas dificuldades no acesso à confirmação laboratorial 6 e pela superposição da sazonalidade do dengue e de outros agravos que fazem parte do seu diagnóstico diferencial 7 , pode-se levantar a hipótese plausível da ocorrência de óbitos não registrados como dengue.

Mediante o exposto, torna-se necessário investigar como o dengue e esses agravos poderiam explicar um possível excesso de óbitos no período epidêmico do dengue.

O conceito de sobremortalidade para detectar o sub-registro de morbidade foi inicialmente explorado por William Farr, ainda no século XIX. Ele definiu excesso de mortalidade como o número de óbitos observados por uma causa que excederam o esperado, em um dado período, em uma determinada comunidade 9,10 .

Essa técnica é usada como parâmetro importante para estimar a gravidade das epidemias 4 , podendo ser útil para o aumento da sensibilidade do sistema de vigilância, uma vez que a cobertura do sistema de mortalidade é mais estável do que o de notificação, e a série histórica disponível, mais longa 11.

O objetivo deste estudo é investigar a ocorrência de sobremortalidade durante a epidemia de dengue ocorrida na Região Metropolitana do Rio de Janeiro entre 2007 e 2008.

\section{Métodos}

Trata-se de um estudo com desenho ecológico de séries temporais. A população de estudo foi constituída de todos os óbitos por dengue e por doenças infecciosas que fazem parte do seu diagnóstico diferencial ocorridos no período de 1986 a 2008, em residentes da Região Metropolitana do Rio de Janeiro. Também foram analisados os casos notificados de dengue em habitantes do Estado do Rio de Janeiro entre 1986 a 2008.

Segundo a Lei Complementar no $105^{12}$, de 4 de julho de 2002, a Região Metropolitana do Estado do Rio de Janeiro é composta por 16 municípios: Belford Roxo, Duque de Caxias, Guapimirim, Itaboraí, Japeri, Magé, Nilópolis, Niterói, Nova Iguaçu, Paracambi, Queimados, Rio de Janeiro, São Gonçalo, São João de Meriti, Seropédica e Tanguá. Entretanto, os municípios de Itaguaí, Mangaratiba, Maricá e Mesquita foram mantidos na presente análise, tendo, em vista, a sua forte relação com a dinâmica metropolitana 13. Essa unidade de análise possui uma área de $5.693,5 \mathrm{~km}^{2}$, equivalente a 13\% de todo o estado (Fundação Centro de Informações e Dados do Rio de Janeiro. Rio de Janeiro em dados. http://www.cide.rj.gov.br/cide/ download/RiodeJaneiroemDados.pdf, acessado em 19/Mai/2008), e uma população total estimada, em 2008, de 11.812.482 habitantes, correspondente a $74,4 \%$ dessa unidade federada (Instituto Brasileiro de Geografia e Estatística. População residente - Rio de Janeiro http://tabnet.datasus. gov.br/cgi/tabcgi.exe?ibge/cnv/popRJ.def, acessado em 10/Fev/2010).

Os dados do SINAN (Superintendência de Vigilância em Saúde. Distribuição de casos de dengue notificados 1986-2008 - mês x ano. http://www. saude.rj.gov.br/Acoes/Dengue_estado.shtml, acessado em 08/Mar/2009) e do SIM (Departamento de Informática do SUS. http://www.datasus.gov.br) foram obtidos da Secretaria de Estado de Saúde do Rio de Janeiro (SES-RJ). Os dados populacionais foram resgatados do Instituto Brasileiro de Geografia e Estatísticas (IBGE. http://www. ibge.gov.br).

As doenças infecciosas consideradas no diagnóstico diferencial do dengue foram: hepatites virais, salmonelose, escarlatina, febre purpúrica 
do Brasil, pneumonia, influenza, enteroviroses, septicemia, infecção meningocócica, leptospirose, riquetsioses, febres recorrentes, malária, arboviroses (febre amarela, Mayaro, Oropouche e outras), hantaviroses, parvoviroses, mononucleose infecciosa, exantema súbito, sarampo, rubéola, citomegalovirose, outras doenças exantemáticas, encefalomielite e outras febres hemorrágicas transmitidas por mosquitos e carrapatos 14 .

Os registros de mortes foram categorizados segundo a 9ạ revisão da Classificação Internacional de Doenças (CID-9) 15, de 1986 a 1995, e por meio da 10a revisão (CID-10) 16, de 1996 até 2008.

Para minimizar as implicações da introdução da CID-10 em análise de tendências da mortalidade por causas, traduziu-se a CID-9 para a CID-10, por meio da conversão manual de arquivos segundo a categoria de três caracteres 17,18.

Os dados de mortalidade foram atualizados em março de 2010, e foram selecionadas as mortes em que estavam registrados os agravos de interesse no campo "causa básica". Além disso, agregaram-se os óbitos de acordo com o mês de ocorrência.

Como se acredita que houve um aumento na gravidade do dengue, com deslocamento dessa para faixas etárias mais jovens da população, especialmente as crianças, realizou-se a estratificação por faixa etária (< 15 anos; $\geq 15$ anos) em todas as análises de mortalidade.

Com o objetivo de identificar quais foram os períodos epidêmicos, foi analisada a série histórica mensal dos casos de dengue ocorridos no Estado do Rio de Janeiro entre 1986 e 2008. Optou-se por utilizar as informações do estado, devido à ausência de dados mensais de alguns municípios da Região Metropolitana do Rio de Janeiro durante o período de interesse.

Devido à falta de consenso sobre o método mais adequado para identificar os meses epidêmicos, arbitrou-se, como sendo o início e término da epidemia, quando houvesse um aumento ou decréscimo de, no mínimo, duas vezes o número de casos do mês de referência em relação ao mês anterior com evidência laboratorial de circulação viral do dengue na comunidade.

Neste estudo, para a predição dos óbitos do ano epidêmico de 2007, um modelo dinâmico Poisson foi ajustado aos dados de mortalidade por dengue e por doenças infecciosas que fazem parte do seu diagnóstico diferencial de 10 de janeiro de 1986 a 31 de dezembro de 2006. Da mesma forma, para obter a predição dos óbitos do ano de 2008, foi considerado o período de 1 o de janeiro de 1986 a 31 de dezembro de 2007.

Toda a inferência estatística foi realizada sob o paradigma bayesiano, que se baseia na distribuição a posteriori do vetor paramétrico, que contém toda a informação probabilística a respeito do parâmetro de interesse.

Insta mencionar que o logaritmo da população foi adicionado ao modelo como variável offset. A interpolação linear foi utilizada para obter o tamanho mensal da população. A estimação para os parâmetros incluiu estimativa pontual e por intervalo, sendo esse um intervalo de $95 \%$ de credibilidade $a$ posteriori.

Os intervalos de credibilidade diferem dos intervalos de confiança gerados pelos métodos tradicionais de inferência estatística. Enquanto o primeiro expressa a probabilidade de que o parâmetro pertença a um intervalo predefinido, condicional aos dados observados 19, o intervalo de confiança quantifica o nível de incerteza envolvido na amostragem 20.

A modelagem foi realizada considerando que $Y_{t}$ é uma variável aleatória que representa o número de óbitos no instante $t, t=1, \ldots, T$. Além disso, supôs-se que:

$Y_{t} \mid \mu_{t} \sim$ Poisson $\left(\mu_{t} N_{t}\right)$

em que $N_{t}$ é conhecido e se refere à população de expostos ao risco de adquirir dengue ou doenças infecciosas que fazem parte do seu diagnóstico diferencial no instante $t$, e $\mu_{t}$ é interpretado como a taxa em que os óbitos ocorrem no tempo $t, t=1$, $\ldots, T$.

Foi possível relacionar um vetor de covariáveis $r_{t}$ ao parâmetro $\mu_{t}$ por meio da relação $\mu_{t}=$ $\lambda_{t} g\left(\boldsymbol{r}_{t}, \boldsymbol{\beta}\right)$, em que $g$ é uma função de ligação, e $\lambda_{t}$ é responsável pela descrição do nível dinâmico. No caso em questão, foi considerado $g\left(\boldsymbol{r}_{\boldsymbol{p}} \boldsymbol{\beta}\right)=\exp \left\{\boldsymbol{r}_{\boldsymbol{t}} \boldsymbol{\beta}^{\boldsymbol{\beta}}\right\}$ e, portanto,

$\mu_{t}=\lambda_{t} \exp \left(\boldsymbol{r}_{\boldsymbol{t}}^{\prime} \boldsymbol{\beta}\right)$

cabe destacar que, neste texto, o negrito foi utilizado para denotar vetores, por exemplo, $\boldsymbol{\beta}=\left(\beta_{1}, \beta_{2}\right.$, $\left.\beta_{3}\right)$ e $\lambda=\left(\lambda_{t}, \ldots, \lambda_{T}\right)$.

Optou-se por ajustar um modelo dinâmico com o objetivo de captar possíveis dependências temporais não explicadas pelas covariáveis utilizadas na análise. Pelo uso de modelos dinâmicos, assumiu-se que o desenvolvimento do sistema em estudo, ao longo do tempo, também pode ser determinado por componentes não observáveis que se relacionam com a série de observações. Assim, o objetivo da análise foi inferir propriedades importantes sobre os componentes não observáveis por meio do conhecimento das observações.

A utilização de modelos dinâmicos e a inclusão de covariáveis que representam características temporais foram uma tentativa de captar estruturas presentes nos dados que modelos lineares generalizados poderiam não captar, já que há uma dependência temporal em séries de natureza epidemiológica, como as de estudo. 
A análise dos dados de forma conjunta para as duas faixas etárias ( $<15$ anos e $\geq 15$ anos) incluindo uma variável indicadora da faixa etária poderia ter sido realizada caso houvesse interesse em quantificar o efeito da faixa etária na série de mortalidade, assumindo o mesmo sistema latente para ambas as faixas. Pela modelagem separada, permitiu-se que o efeito dinâmico latente estimado fosse distinto para cada faixa etária.

Considerou-se que

$\mu_{t}=\lambda_{t} \exp \left\{r_{t, 1} \beta_{1}+r_{\mathrm{t}, 2} \beta_{2}+r_{\mathrm{t}, 3} \beta_{3}\right\}$

em que $r_{t, 1}$ é uma variável dummy que indica os períodos epidêmicos de dengue, e $r_{t, 2}$ e $r_{t, 3}$ correspondem às componentes sazonais sen $(2 \pi t / 12)$ e $\cos (2 \pi t / 12)$, para $t=1,2, \ldots, T$, respectivamente.

As componentes sazonais foram incluídas, pois a incidência do dengue é influenciada pelas condições climáticas, que variam dependendo da estação do ano. Da mesma forma, foi inserida a variável indicadora dos períodos de epidemia de dengue, pois se acredita que haja um aumento no número de óbitos durante os períodos epidêmicos. Portanto, por meio da componente $\beta_{1}$, correspondente à variável indicadora, mensurou-se o efeito da ocorrência de epidemia na série de óbitos em análise.

A dinâmica do nível $\lambda_{t}$ foi dada pela seguinte equação de evolução:

$\lambda=\frac{\lambda_{t-1} \eta_{t}}{\omega}$

em que:

$n_{\mathrm{t}} \sim \operatorname{Beta}\left(\omega \alpha_{\{t-1\}},(1-\omega) a_{t-1}\right)$

sendo $a_{\mathrm{t}}$ um parâmetro da distribuição $a$ posteriori de $\lambda_{t} \mid D_{t}$, em que $D_{t}$ denota o conjunto de informação no tempo $t, \mathrm{t}=1,2, \ldots, T$.

Uma descrição mais detalhada sobre essa família de modelos dinâmicos, na qual esse modelo foi baseado, pode ser visto em Franco et al. 20. Na literatura, Harvey \& Fernandes 21 tratam desses modelos de séries temporais para dados de contagem em que a dinâmica do nível é inicializada com uma especificação a priori conjugada.

Nesse caso, verificou-se que $\log \left(\lambda_{t}\right)=\log \left(\lambda_{t-1}\right)+$ $n_{t}^{*}$, em que $n_{t}^{*}=\log \left(\frac{n_{t}}{\omega}\right)$. O parâmetro $\omega$, que varia entre 0 e 1, desempenha um papel de aumentar a variância do sistema latente, que é caracterizado pelos parâmetros $\lambda$, devido à passagem de tempo.

Note que

$\operatorname{Var}\left(\lambda_{t}, D_{t-1}\right)=\omega^{-1} \frac{a_{t-1}}{b_{t-1}^{2}}=\omega^{-1} \operatorname{Var}\left(\lambda_{t-1}, D_{t-1}\right)$.

Pensando-se em termos da precisão (inverso da variância), na passagem do tempo entre t-1 e t, apenas $100 \omega \%$ da informação é preservada.

Para completar a especificação do modelo, considerou-se a seguinte distribuição $a$ priori: 22 $p(\lambda, \beta, \omega)=p(\lambda \mid \beta, \omega) p(\beta) p(\omega)$

em que foi assumido que:

$\left[\left(\begin{array}{l}\beta_{1} \\ \beta_{2} \\ \beta_{3}\end{array}\right) \sim N\left(\begin{array}{l}0 \\ 0 \\ 0\end{array}\right),\left(\begin{array}{lll}\mathrm{c} & 0 & 0 \\ 0 & \mathrm{c} & 0 \\ 0 & 0 & \mathrm{c}\end{array}\right)\right]$

$\omega \sim U(0,1]$

$\lambda_{1} \mid \omega, D_{0} \sim \operatorname{Gama}\left(\omega a_{0}, \omega b_{0}\right)$

Nessa aplicação, foram consideradas as seguintes especificações $a$ priori: $c=1.000, a_{0}=$ 0,01 e $b_{0}=0,01$. A distribuição uniforme entre zero e um foi escolhida devido à sua estrutura, já que, a priori, o mesmo peso é dado para todos os valores pertencentes ao intervalo $U(0,1]$. Essas especificações levam a distribuições $a$ priori marginais não informativas para cada um dos parâmetros do modelo. Optou-se pelo uso de distribuições $a$ priori não informativas para $\boldsymbol{\beta}, \omega \mathrm{e}$ $\lambda_{1} \mid \omega, D_{0}$ a fim de se caracterizar, na análise, a falta de informação a priori a respeito desses parâmetros. Com isso, a posteriori, espera-se que a informação vinda dos dados tenha mais influência ao se comparar com a informação a priori.

Fato importante de se esclarecer é a característica hierárquica do modelo assumido. A distribuição Gama para $\lambda_{1} \mid \omega, D_{0}$ é uma suposição importante, já que, por consequência, as equações de evolução possuem forma analítica fechada. Caso a estrutura matemática da distribuição $a$ priori $\lambda_{1} \mid \omega, D_{0}$ fosse diferente, métodos de aproximação para obter a distribuição $a$ posteriori de $\left(\lambda_{1}, \ldots, \lambda_{T} \boldsymbol{\beta}, \omega, D_{T}\right)$ seriam necessários. Por outro lado, o usuário tem uma flexibilidade de escolha da estrutura matemática da distribuição $a$ priori para os hiperparâmetros $\boldsymbol{\beta}$ e $\omega$.

Todo o procedimento foi realizado sob abordagem bayesiana. Dificuldades surgem já que a distribuição a posteriori $p\left(\lambda, \beta_{1}, \beta_{2}, \beta_{3}, \omega \mid y\right)$ não pode ser obtida analiticamente. Assim, métodos de Monte Carlo via Cadeias de Markov são utilizados para gerar amostras da distribuição $a$ posteriori 23 .

Em relação à inferência para os parâmetros estáticos, a distribuição a posteriori de $\varphi=(\boldsymbol{\beta}, \omega)$ é:

$$
p(\beta, \omega ; y)=\frac{l(\boldsymbol{\beta}, \omega ; \boldsymbol{y}) p(\boldsymbol{\beta}) p(\omega)}{\int l(\boldsymbol{\beta}, \omega ; \boldsymbol{y}) p(\boldsymbol{\beta}) p(\omega) d \beta d \omega}
$$

A verossimilhança marginal é dada por $l(\boldsymbol{\beta}, \omega ; y)=\prod_{t=1}^{T} p\left(y_{t} \boldsymbol{\beta}, \omega, D_{t-1}\right)$, em que a distribuição preditiva $p\left(y_{t} \mid \boldsymbol{\beta}, \omega, D_{t-1}\right)$ pertence a uma distribuição binomial negativa, o que pode ser visto resolvendo a seguinte integral:

$$
p\left(y_{t} \mid \boldsymbol{\beta}, \omega, D_{t-1}\right)=\int \underbrace{p\left(y_{t} \mid \lambda_{t}, \beta\right)}_{\text {Poisson }} \underbrace{p\left(\lambda_{t} \beta, \omega, D_{t-1}\right)}_{\text {Gama }} d \lambda_{t}
$$


Nesse caso, a distribuição a posteriori não está disponível em forma fechada, portanto, são utilizados os métodos Monte Carlo via Cadeias de Markov para aproximar $p(\boldsymbol{\beta}, \omega ; \boldsymbol{y})$.

Quanto à inferência para os parâmetros de estado, considere os seguintes resultados 20 , condicionalmente a $\varphi=(\boldsymbol{\beta}, \omega)$, embora não esteja explícito na notação:

a) A distribuição a priori $\lambda_{t} \mid D_{t-1}$ segue uma distribuição Gama $\left(a_{t \mid t-1}, b_{t \mid t-1}\right)$, em que $a_{t \mid t-1}=\omega a_{t-1} \mathrm{e}$ $b_{t \mid t-1}=\omega b_{t-1}$.

b) A distribuição a posteriori $\lambda_{t} \mid D_{t}$ segue uma distribuição Gama $\left(a_{t}^{*}, b_{t}^{*}\right)$, em que

$a_{t}^{*}=a_{t \mid \mathrm{t}-1}+y_{t}$ e $b_{t}^{*}=b_{t \mid t-1}+N_{t} e^{r_{t} \boldsymbol{\beta}}$.

A demonstração desses resultados pode ser encontrada em Santos 22.

Consequentemente, $\lambda_{t-1}-\omega \lambda_{t} \mid \lambda_{t}, D_{t-1} \sim$ Gama $\left((1-\omega) a_{t-1}^{*}, b_{t-1}^{*}\right)$, o que segue diretamente do resultado 1.

Note que:

$p\left(\lambda_{1}, \ldots, \lambda_{T} D_{T}\right)=p\left(\lambda_{T} D_{T}\right) \Pi_{t=1}^{T-1} p\left(\lambda_{t} \mid \lambda_{t+1}, D_{T}\right)$

E que $p\left(\lambda_{t} \mid \lambda_{t+1}, D_{t}\right)$ é conhecido. Com isso, é possível construir amostras de $\left(\lambda_{1}, \ldots, \lambda_{T} \mid D_{t}\right)$ baseado no seguinte algoritmo:

a) Faça $t=T$ e amostre de $p\left(\lambda_{t} \mid D_{T}\right)$;

b) Faça $t \leftarrow t$-1 e amostre de $p\left(\lambda_{t} \mid \lambda_{t+1}, D_{t}\right)$;

c) Se $t>1$, retorne ao item 2. Caso contrário, a amostra de $\left(\lambda_{1}, \ldots, \lambda_{T} \mid D_{t}\right)$ está completa.

Para serem feitas previsões, para $h=1,2, \ldots$, a densidade $y_{T+h} \mid D_{T}$ é obtida por meio de:

$p\left(y_{T+h} \mid D_{T}\right)=\int p\left(y_{T+h} \mid \varphi, \lambda, D_{T}\right) p\left(\varphi, \lambda \mid D_{T}\right) d \varphi d \lambda$.

Essa distribuição pode ser aproximada por:

$p\left(y_{T+h} \mid D_{T}\right) \approx \frac{1}{L} \sum_{i=1}^{L} p\left(y_{T+h} \mid \varphi^{(i)}, \lambda^{(i)}, D_{T}\right)$,

em que L é referente ao número de simulações do algoritmo Monte Carlo via Cadeias de Markov, sendo $\varphi^{(\mathrm{i})}$ e $\lambda^{(\mathrm{i})}$ gerados pela distribuição $a$ posteriori $p\left(\varphi, \lambda \mid D_{T}\right)$.

$\mathrm{O}$ excesso de mortalidade foi calculado pela diferença do número de mortes ocorridas nos meses dos anos de 2007 e 2008 e o número de óbitos esperados com base em uma experiência sazonal anterior 9,10 .

Foram utilizados, para análise e processamento dos dados, os softwares Microsoft Excel 2010 (Microsoft Corp., Estados Unidos), Tabwin versão 3.6b (Departamento de Informática do SUS. http://www2.datasus.gov.br/DATASUS/index. php?area $=040805$ \&item $=3$ ) e o R (The R Foundation for Statistical Computing, Viena, Áustria; http://www.r-project.org). O projeto foi aprovado pela Comissão de Ética e Pesquisa do Instituto de Estudos em Saúde Coletiva, da Universidade Federal do Rio de Janeiro (parecer no 87/2009, processo no 19/2009).

\section{Resultados}

Nos anos de 2007 e 2008, o número de casos de dengue mensais variou de 663 a 90.945 . Durante esse período, o pico de casos foi nos meses de março e abril de 2008, com 86.034 e 90.945 casos, respectivamente, conforme é demonstrado na Tabela 1, que apresenta a distribuição dos casos notificados e dos períodos epidêmicos de dengue no Estado do Rio de Janeiro de 1986 a 2008, por mês de ocorrência.

Na Figura 1, encontram-se os histogramas para as amostras finais de cada parâmetro estático do modelo dinâmico Poisson ajustado aos dados de óbitos por dengue e por doenças infecciosas que fazem parte do seu diagnóstico diferencial ocorridos em residentes da Região Metropolitana do Rio de Janeiro, entre lo de janeiro de 1986 a 31 de dezembro de 2006, por faixa etária. Da mesma forma, na Figura 2, observam-se os histogramas referentes ao período de 1o de janeiro de 1986 a 31 de dezembro de 2007.

Verificou-se que o valor da mediana do parâmetro $\beta_{1}$ relacionado à variável indicadora de epidemia foi positivo para todos os cenários. Isso pode indicar que a presença de epidemia levou a um aumento de óbitos. Entretanto, para esse conjunto de dados, essa componente não foi significativa ao nível de 95\% de credibilidade, já que o parâmetro só possui uma única mediana.

Na Figura 3, observa-se uma tendência decrescente do nível $\mathrm{N}_{t} \mathrm{\mu}_{t}$. Estimou-se que, em média, os óbitos se reduziram com o passar do tempo. Para os indivíduos < 15 anos, o nível estimado foi inferior àqueles $\geq 15$ anos. Além disso, para os indivíduos $\geq 15$ anos, o nível estimado é mais errático, e há mais incerteza na estimação. A componente $\beta_{2}$ não se mostrou significativa.

Apesar de o intervalo de credibilidade dos parâmetros $\beta_{1}$, referente à variável indicadora de epidemia, e $\beta_{2}$, referente ao componente sazonal, ter incluído o zero, essas covariáveis permaneceram no modelo devido a sua importância epidemiológica, já que a dengue é uma doença sazonal que evoluiu no período de estudo para distintos processos epidêmicos.

Na Figura 4, estão disponíveis os óbitos mensais observados e esperados por dengue e por doenças infecciosas que fazem parte do seu diagnóstico diferencial em residentes da Região Metropolitana do Rio de Janeiro, nos anos de 2007 e 2008, por faixa etária. Em 2007, apesar de, na maioria dos meses, os valores observados estarem acima da mediana dos óbitos esperados, em ambas as faixas etárias, os óbitos observados não ultrapassaram o limite superior do intervalo de $95 \%$ de credibilidade a posteriori. Em 2008, verificou-se comportamento similar nos indivíduos com 15 
Tabela 1

Distribuição dos casos notificados e dos períodos epidêmicos de dengue no Estado do Rio de Janeiro, Brasil, de 1986 a 2008 , por mês de ocorrência.

\begin{tabular}{|c|c|c|c|c|c|c|c|c|c|c|c|c|c|}
\hline \multirow[t]{2}{*}{ Ano } & \multicolumn{12}{|c|}{ Mês } & \multirow[t]{2}{*}{ Total } \\
\hline & 1 & 2 & 3 & 4 & 5 & 6 & 7 & 8 & 9 & 10 & 11 & 12 & \\
\hline 1987 & 14.074 * & 16.422 * & 15.217 * & 8.182 * & 3.696 * & 1.129 * & 351 * & 58 & 22 & 21 & 74 & 109 & 59.355 \\
\hline 1988 & 302 & 205 & 297 & 143 & 116 & 86 & 31 & 77 & 48 & 20 & 67 & 58 & 1450 \\
\hline 1989 & 123 & 99 & 93 & 213 & 160 & 88 & 48 & 19 & 13 & 23 & 83 & 182 & 1144 \\
\hline 1990 & 55 & 44 & 104 & 1.140 * & 2.423 * & 2.383 * & 2.341 * & 779 & 406 & 399 & 2.798 * & 6.813 * & 19.685 \\
\hline 1991 & 34.636 * & 20.036 * & 14.783 * & 9.903 * & 3.565 * & 1.290 * & 403 & 217 & 249 & 191 & 242 & 376 & 85.891 \\
\hline 1992 & 437 & 357 & 244 & 91 & 90 & 80 & 47 & 38 & 39 & 66 & 91 & 78 & 1658 \\
\hline 1993 & 62 & 50 & 300 & 43 & 18 & 8 & 9 & 17 & 24 & 20 & 34 & 38 & 623 \\
\hline 1994 & 28 & 23 & 77 & 14 & 20 & 23 & 34 & 20 & 16 & 23 & 6 & 3 & 287 \\
\hline 1995 & 586 * & 7.817 * & 16.442 * & 7.743 * & 2.235 * & 124 & 85 & 65 & 21 & 20 & 53 & 49 & 35.240 \\
\hline 1997 & 308 & 370 & 289 & 158 & 100 & 75 & 43 & 37 & 47 & 28 & 67 & 782 * & 2.304 \\
\hline 1998 & 1.554 * & 1.917 * & 8.272 * & 11.359 * & 2.950 * & 528 * & 154 & 85 & 55 & 57 & 122 & 5.329 * & 32.382 \\
\hline 1999 & 667 * & 835 * & 1.770 * & 2.437 * & 1.459 * & 319 * & 137 & 113 & 96 & 83 & 63 & 1.104 * & 9.083 \\
\hline 2000 & 289 * & 318 * & 612 * & 923 * & 1.003 * & 392 * & 199 & 150 & 102 & 95 & 96 & 102 & 4.281 \\
\hline 2001 & 3.055 * & 7.435 * & 11.086 * & 19.287 * & 20.596 * & 8.530 * & 3.386 * & 1.303 * & 657 & 931 & 926 & 3.023 * & 80.215 \\
\hline 2002 & 49.280 * & 93.016 * & 99.861 * & 31.642 * & 7.208 * & 1.672 & 985 & 502 & 272 & 522 & 1.522 * & 1.763 * & 288.245 \\
\hline 2003 & 1.797 * & 2.390 * & $1.755 *$ & 1.000 * & 545 * & 263 & 140 & 100 & 104 & 159 & 415 & 574 & 9.242 \\
\hline 2004 & 508 & 341 & 443 & 211 & 132 & 76 & 84 & 112 & 128 & 159 & 192 & 308 & 2.694 \\
\hline 2005 & 358 & 256 & 218 & 167 & 126 & 157 & 115 & 191 & 146 & 189 & 267 & 390 & 2.580 \\
\hline 2006 & 1.920 * & 4.515 * & 8.036 * & 7.685 * & 4.153 * & 1.355 * & 626 & 841 & 326 & 337 & 423 & 837 & 31.054 \\
\hline 2007 & 3.229 * & 6.261 * & 14.225 * & 15.019 * & 11.050 * & 4.452 * & 2.293 * & 1.085 & 847 & 1.262 & 2.777 * & 4.053 * & 66.553 \\
\hline 2008 & 17.604 * & 25.319 * & 86.0348 & 90.945 * & 24.5438 & 4.481 * & 1.495 & 875 & 663 & 945 & 1.421 & 1.493 & 255.818 \\
\hline
\end{tabular}

* Meses epidêmicos de dengue.

Fonte: Superintendência de Vigilância em Saúde, Secretaria de Estado de Saúde do Rio de Janeiro. Distribuição de casos de dengue notificados $1986-2008$ mês x ano. http://www.saude.rj.gov.br/Acoes/Dengue_estado.shtml (acessado em 08/Mar/2009).

anos ou mais de idade. Entretanto, em menores de 15 anos de idade, foram encontrados quatro óbitos acima do limite superior do intervalo de $95 \%$ de credibilidade a posteriori no mês de março.

\section{Discussão}

Os resultados obtidos apontam para a ocorrência de sobremortalidade em residentes da Região Metropolitana do Rio de Janeiro, menores de 15 anos de idade, em março de 2008 e para a possibilidade de sua detecção por meio de dados mensais de óbitos.

Estudo anterior que investigou a ocorrência de sobremortalidade por todos os óbitos exceto por causas externas, na Região Metropolitana do Rio de Janeiro, durante a epidemia de dengue de 20012002, também identificou excesso de óbitos. Entretanto, ao comparar as faixas etárias com "menos de 60 anos de idade" e com "60 anos ou mais de idade", detectou-se sobremortalidade, em janeiro e março de 2002, somente na população idosa 4 .

Tem sido discutido, em estudos que avaliam o excesso de óbitos durante epidemias de influenza, que a mortalidade por todas as causas poderia ser um melhor indicador da carga total dessa doença que a mortalidade por pneumonia e influenza. Uma justificativa apresentada baseia-se em um estudo realizado por Glezen 24, em 1982, que verificou que, para cada morte por influenza registrada oficialmente como pneumonia, existiam três óbitos que não eram identificados. Além disso, discute-se que a causa de morte normalmente não está confirmada laboratorialmente, podendo ser equivocada, uma vez que a forma de classificá-la é subjetiva 9 .

Por outro lado, é incorreto afirmar que todos os óbitos ocorridos durante uma epidemia são devidos a esse agravo. Os óbitos por outras doenças 
Figura 1

Histogramas para as amostras finais de cada parâmetro do modelo dinâmico Poisson ajustado aos dados de óbitos por dengue e por outras doenças infecciosas em residentes da Região Metropolitana do Rio de Janeiro, Brasil, entre 1986 e 2006, por faixa etária.

1a) Menor que 15 anos, $\beta_{1}$

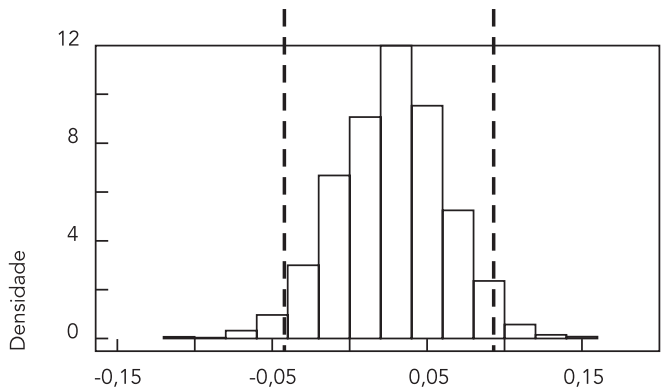

1c) Menor que 15 anos, $\beta_{2}$

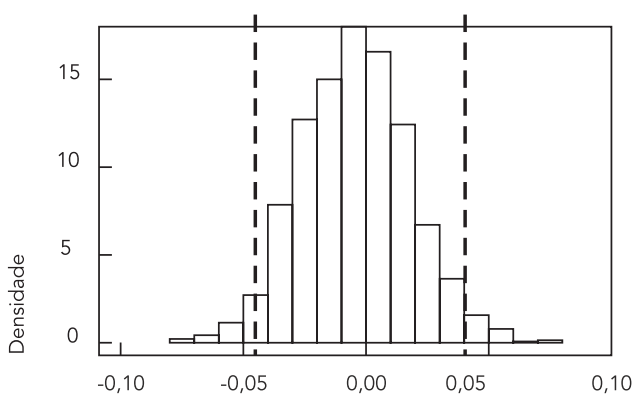

1e) Menor que 15 anos, $\beta_{3}$

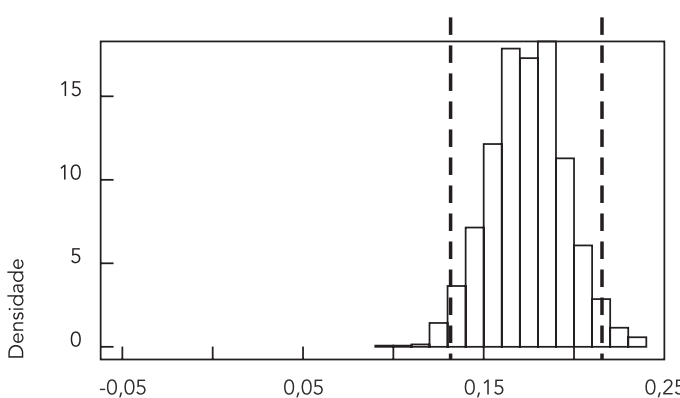

1b) 15 anos ou mais, $\beta_{1}$

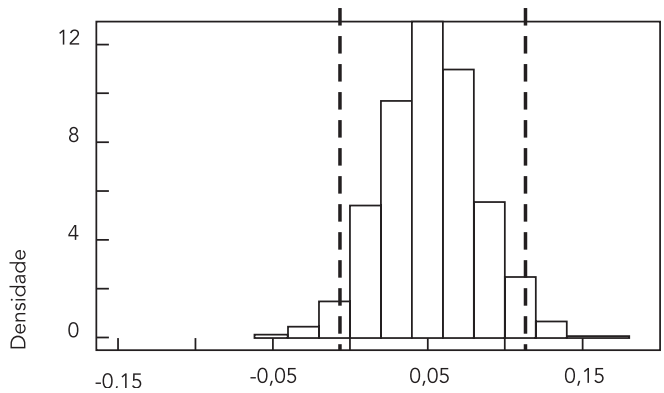

1d) 15 anos ou mais, $\beta_{2}$

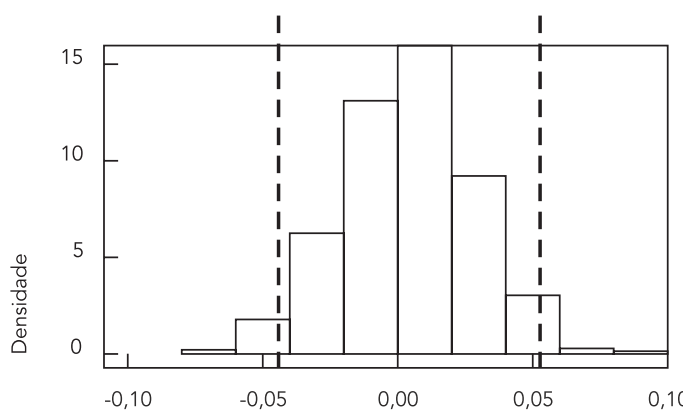

1f) 15 anos ou mais, $\beta_{3}$

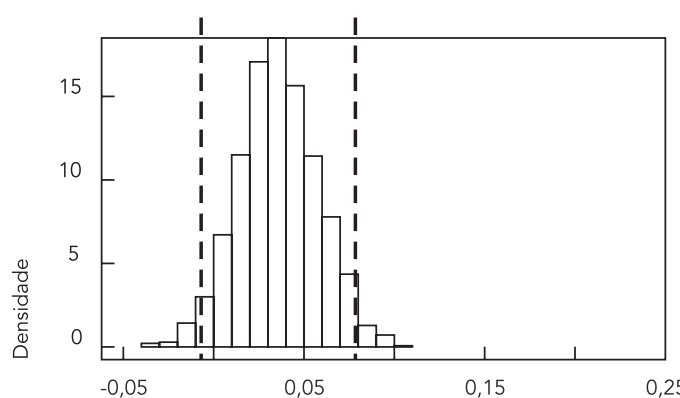


1g) Menor que 15 anos, $\omega$

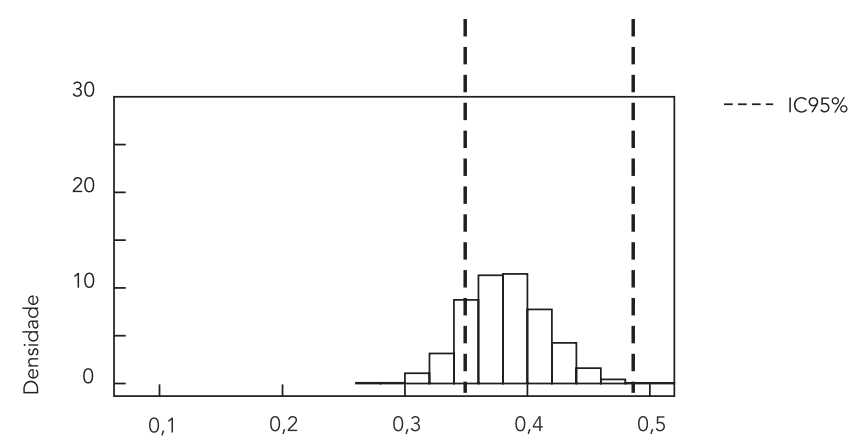

1h) 15 anos ou mais, $\omega$

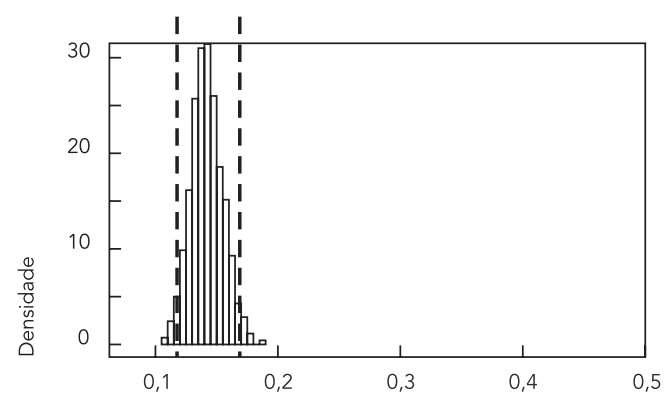

Nota: as linhas pontilhadas referem-se ao intervalo de $95 \%$ de credibilidade. O $\beta_{1}$ é a componente relacionada à variável indicadora de epidemiologia. O $\beta_{2}$ e o $\beta_{3}$ são as componentes sazonais. $\bigcirc \omega$ é o parâmetro que possui o papel de aumentar a variância devido à passagem do tempo.

Figura 2

Histogramas para as amostras finais de cada parâmetro do modelo dinâmico Poisson ajustado aos dados de óbitos por dengue e por outras doenças infecciosas em residentes da Região Metropolitana do Rio de Janeiro, Brasil, entre 1986 e 2007, por faixa etária.

2a) Menor que 15 anos, $\beta_{1}$

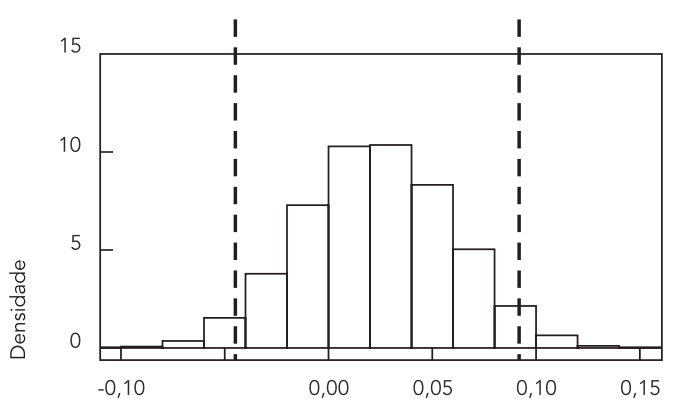

2c) Menor que 15 anos, $\beta_{2}$

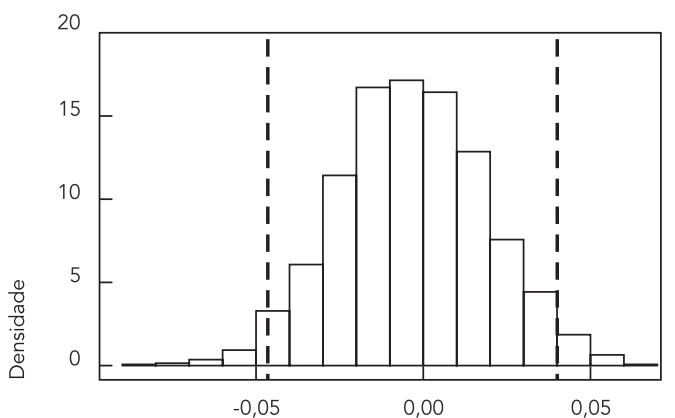

2b) 15 anos ou mais, $\beta_{1}$

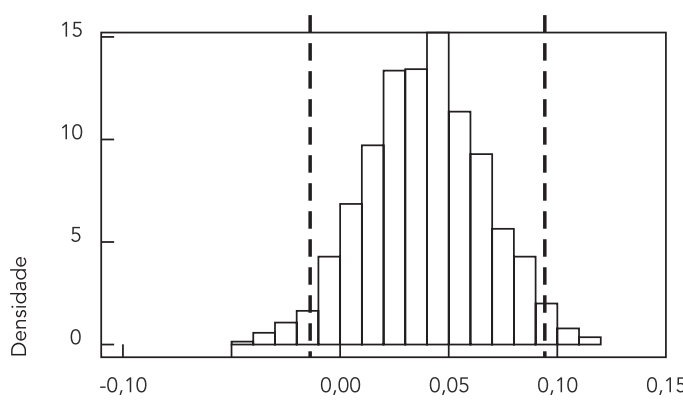

2d) 15 anos ou mais, $\beta_{2}$

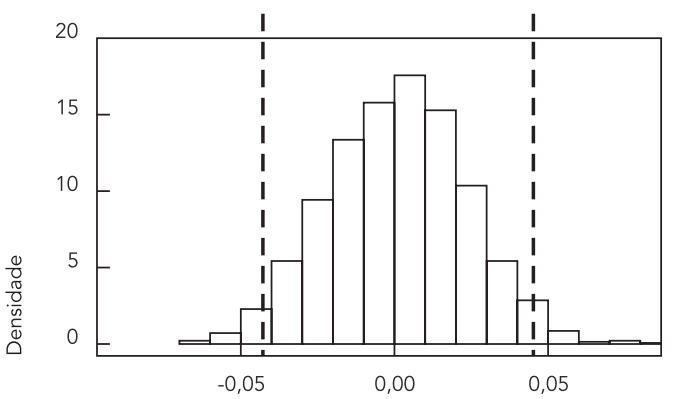

--- IC95\%

(continua) 
2e) Menor que 15 anos, $\beta_{3}$

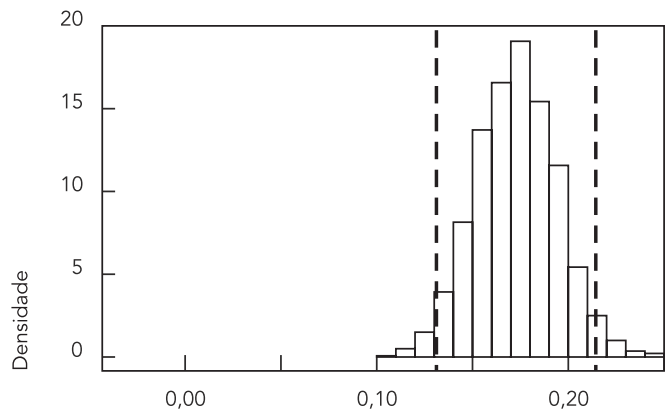

2g) Menor que 15 anos, $\omega$

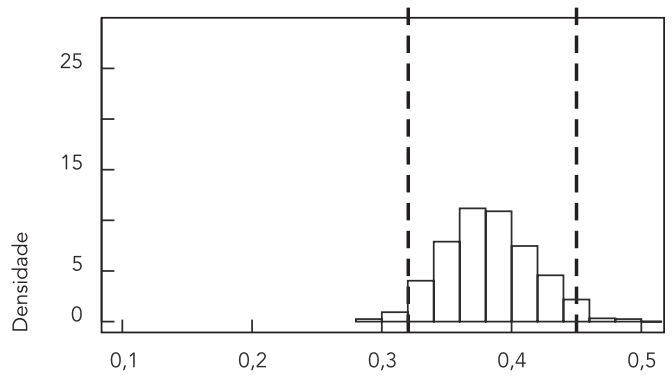

2f) 15 anos ou mais, $\beta_{3}$

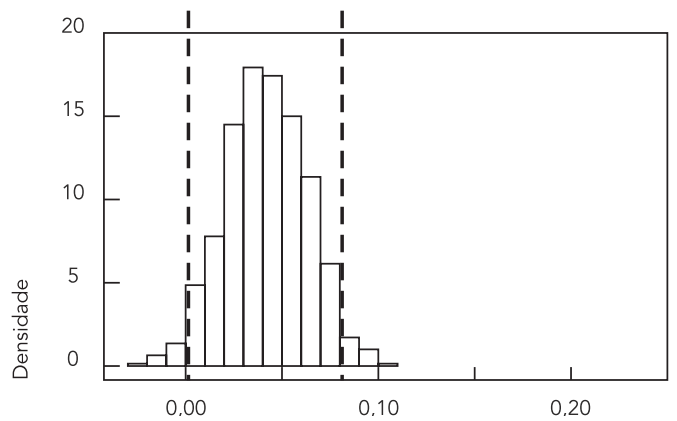

2h) 15 anos ou mais, $\omega$

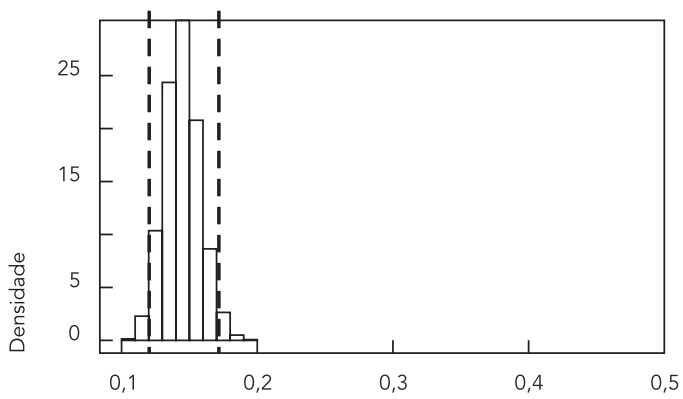

Nota: as linhas pontilhadas referem-se ao intervalo de $95 \%$ de credibilidade. $\bigcirc \beta_{1}$ é a componente relacionada à variável indicadora de epidemiologia. $\bigcirc \beta_{2}$ e o $\beta_{3}$ são as componentes sazonais. $\bigcirc \omega$ é o parâmetro que possui o papel de aumentar a variância devido à passagem do tempo.

podem aumentar durante semanas nas quais a epidemia está presente 25 . Da mesma forma, tem se argumentado que as estimativas de mortalidade sazonal por todas as causas carecem de precisão ${ }^{9}$.

Nesta investigação, foram analisados os óbitos por dengue e por doenças infecciosas do seu diagnóstico diferencial, pois alguns inquéritos têm demonstrado baixa confiabilidade dos dados de mortalidade por dengue 6 e dificuldades no diagnóstico desse agravo, tanto pela sintomatologia inespecífica e similaridade do quadro clínico com outras doenças infecciosas 5,6 quanto pela superposição da sazonalidade desses agravos 5 e dificuldades no acesso à confirmação laboratorial 6 .

Considerando que o aumento na mortalidade deve ser precedido por um incremento na morbidade ${ }^{26}$, pode-se supor que, em março de 2008 , houve um excesso de mortalidade por dengue em crianças menores de 15 anos de idade que residiam na Região Metropolitana do Rio de Janeiro.

Outros fatores que reforçam essa hipótese são: a dificuldade no diagnóstico precoce dos casos de febre hemorrágica do dengue ${ }^{8}$, o elevado número de internações por dengue em crianças menores de 15 anos de idade (Superintendência de Vigilância em Saúde. Distribuição de casos de dengue notificados 1986-2008 - mês x ano. http://www.sau de.rj.gov.br/Acoes/Dengue_estado.shtml, acessado em 08/Mar/2009) e de indivíduos suscetíveis à doença e a desestruturação dos serviços básicos de saúde 27 .

Além disso, na infância, é difícil diferenciar o dengue de outras doenças febris agudas, e a maioria das infecções por dengue é assintomática ou oligossintomática 28,29 . Um inquérito realizado no Rio de Janeiro verificou sorologia positiva para dengue em $33 \%$ dos escolares, apesar de os pais informarem que eles não tinham tido a doença ${ }^{30}$. Pesquisa com crianças tailandesas com rubor facial e febre indiferenciada de curta duração diagnosticou dengue em $35 \%$ dos casos 31 . Em uma investigação anterior realizada em período epidêmico, essa taxa foi de $90 \% 32$. No Paquistão, a frequência de dengue em indivíduos entre 4 e 9 
Figura 3

Estimativas a posteriori da média de óbitos por dengue e por outras doenças infecciosas em residentes da Região Metropolitana do Rio de Janeiro, Brasil, entre 1986 a 2006 e 1986 a 2007, por faixa etária, com seus respectivos intervalos de 95\% de credibilidade (IC95\%).

3a) Menor que 15 anos, 1986-2006

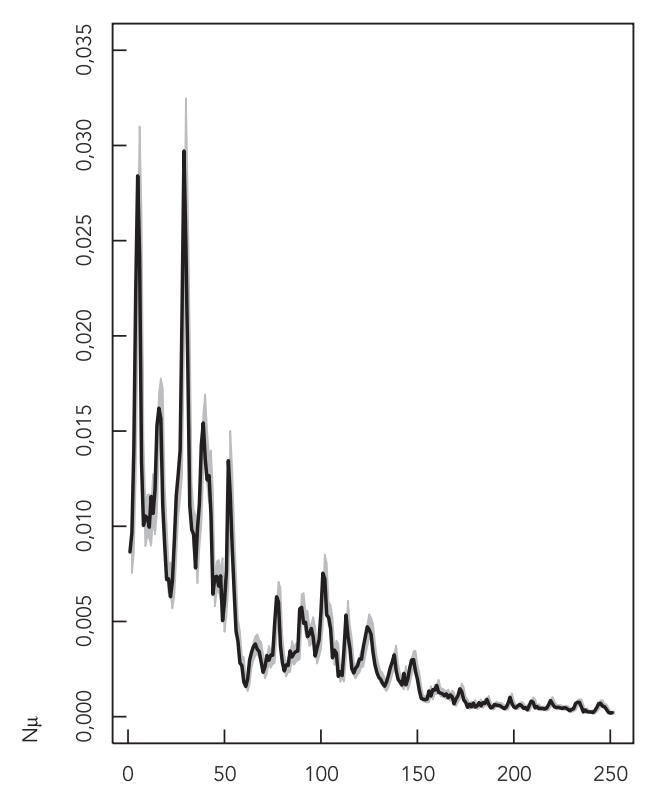

3c) Menor que 15 anos, 1986-2007

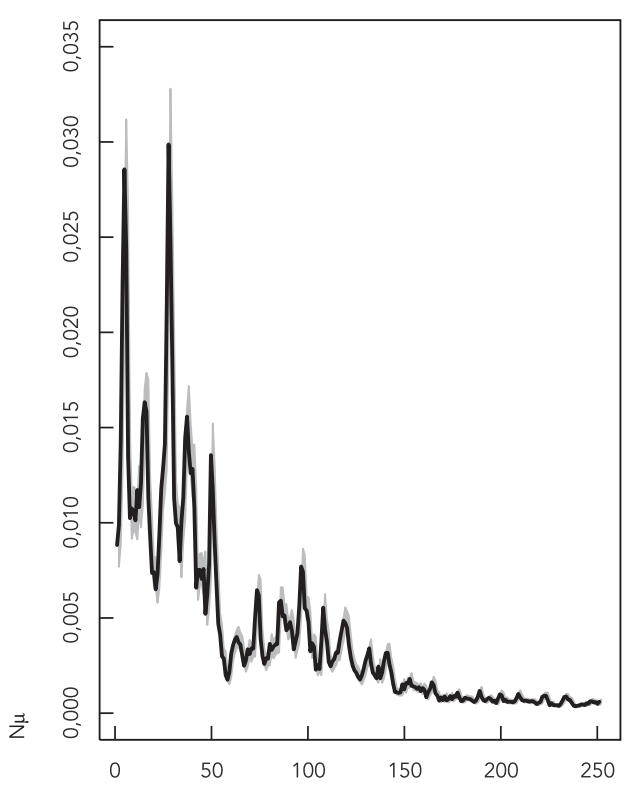

3b) 15 anos ou mais, 1986-2006

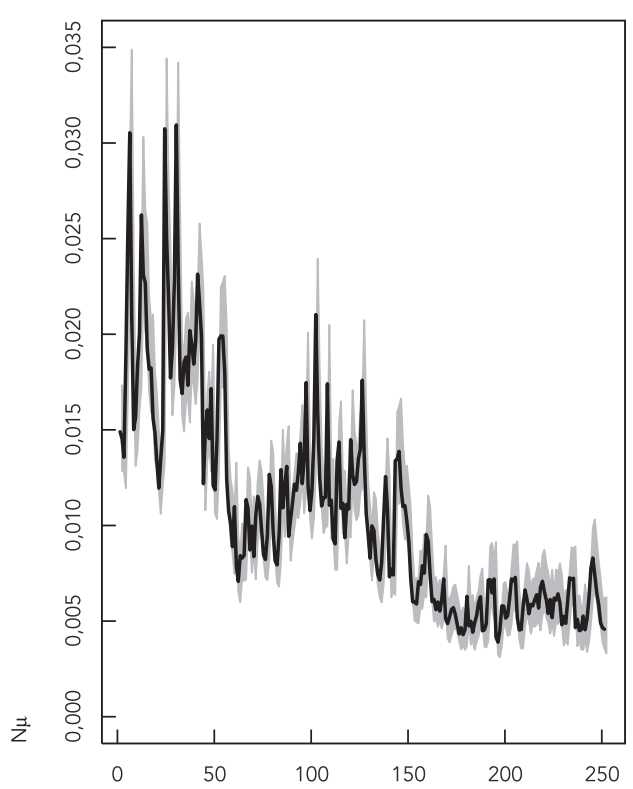

3d) 15 anos ou mais, $1986-2007$

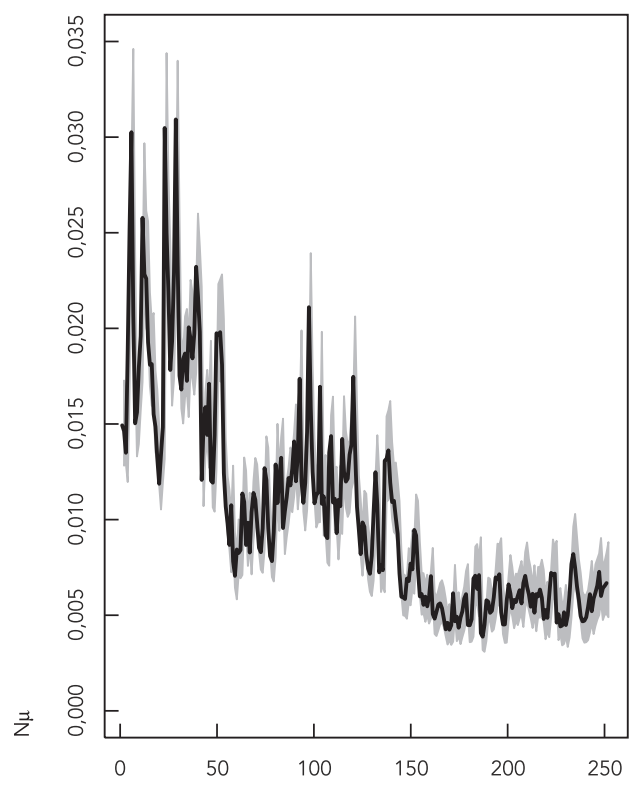


Figura 4

Óbitos mensais por dengue e por outras doenças infecciosas, observados e esperados, em residentes da Região Metropolitana do Rio de Janeiro, Brasil, nos anos de 2007 e 2008, por faixa etária.

4a) Menor que 15 anos, 2007

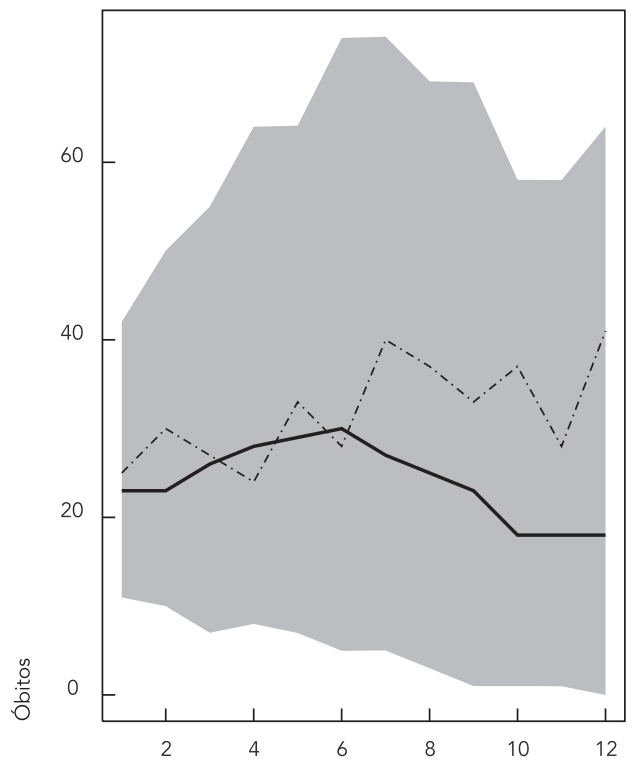

4c) Menor que 15 anos, 2008

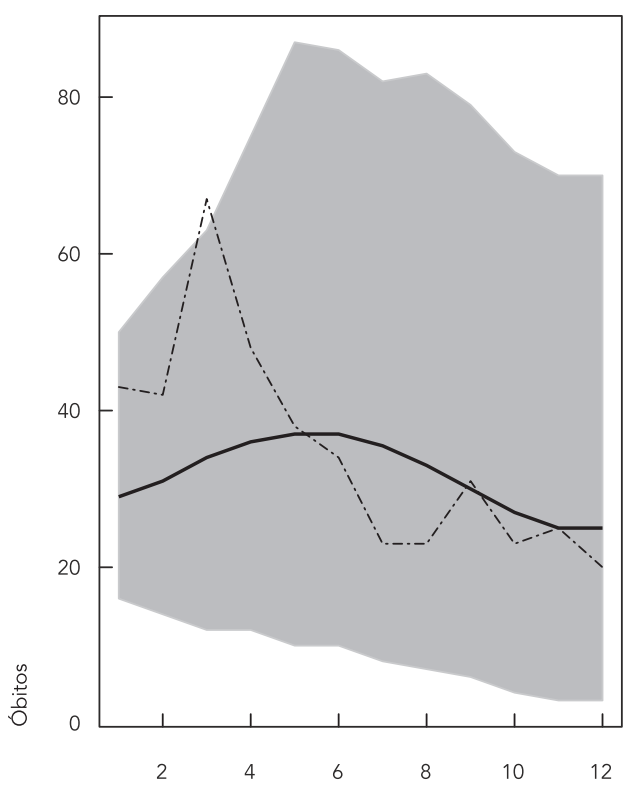

4b) 15 anos ou mais, 2007
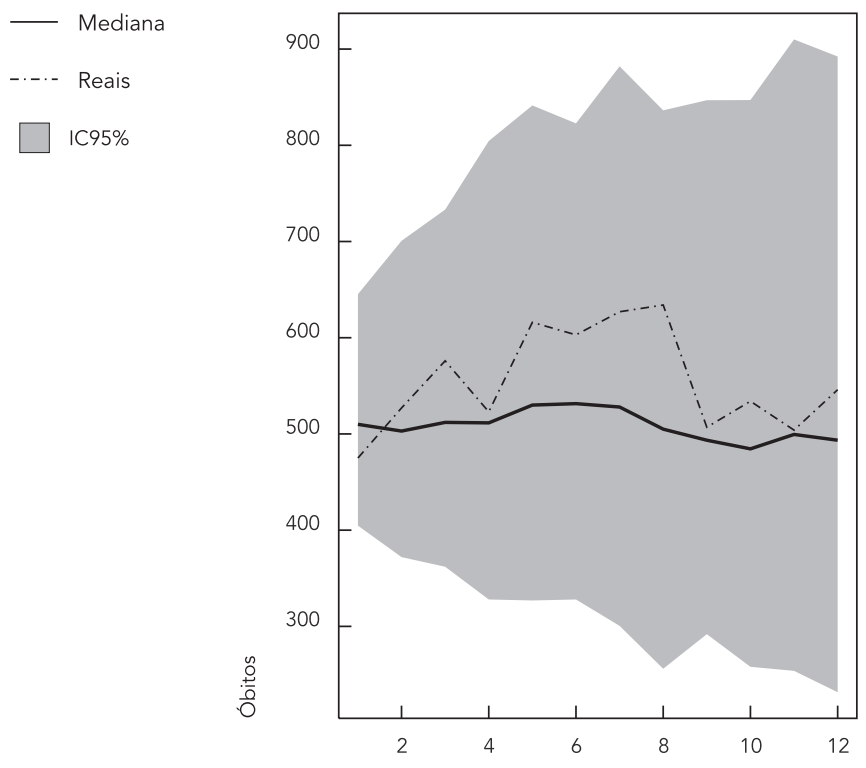

- Mediana

-.... Reais

$\square \quad 1 \mathrm{C} 95 \%$

4d) 15 anos ou mais, 2008

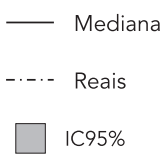

- Mediana

-...- Reais

IC95\% 
anos de idade, com febre de curta duração sem etiologia definida foi de $26 \% 33$.

Também pode ser destacada a dificuldade no diagnóstico de dengue com base no critério de definição de caso suspeito de dengue preconizado pelo Ministério da Saúde e descrito a seguir: paciente com febre com duração máxima de sete dias, acompanhada de, pelo menos, dois dos seguintes sintomas: cefaleia, dor retro-orbital, mialgia, artralgia, prostração, exantema, e com exposição à área com transmissão de dengue ou com presença de Aedes aegypti nos últimos 15 dias 14. Em um estudo transversal realizado em uma unidade hospitalar de Minas Gerais, Brasil, com pacientes de até 12 anos de idade apresentando doença febril aguda sem etiologia definida, identificou que, se o diagnóstico fosse baseado nesse critério, quase metade das crianças com dengue não teriam sido identificadas 28 .

Outro resultado que merece atenção e que pode ser um objeto interessante de investigação é a redução do número de óbitos por dengue e por doenças infecciosas que fazem parte do seu diagnóstico diferencial em abril de 2008, mês com maior número de casos de dengue do período de 2007-2008.

Talvez a redução do número de óbitos seja um reflexo da intensificação das ações de controle do dengue que foram iniciadas no final de março de 2008. Nesse momento, foi instalado um Gabinete de Crise, integrado pelos três níveis de governo, em parceria com as Forças Armadas e a Defesa Civil. Dentre as atividades que foram desencadeadas, destacam-se: o mapeamento e a organização das ações de atenção à saúde da população segundo um novo fluxograma para o atendimento dos pacientes com suspeita de dengue; a ampliação do horário de funcionamento dos postos de saúde do Município do Rio de Janeiro, que passaram a funcionar 24 horas por dia; a contratação de pessoal em caráter emergencial; a expansão de leitos em hospitais federais, estaduais e municipais e na rede privada contratada do SUS; a criação de uma central de regulação de leitos de dengue com o objetivo de coordenar a reserva dos leitos para os casos graves; a implantação de centros de hidratação oral ou venosa e de postos de triagem dos doentes; a montagem de um sistema de transporte de pacientes; o estímulo à doação de sangue, com implantação de ônibus de coleta volante; as campanhas de mobilização da sociedade no combate ao mosquito; a manutenção dos bombeiros no combate aos criadouros; os mutirões de combate aos focos, o treinamento dos agentes de saúde para operarem as máquinas de desinfestação 27 e a ampla divulgação de informações sobre a doença na mídia.

\section{Conclusão}

A partir deste estudo, pode-se levantar a hipótese de que houve excesso de mortalidade por dengue e por doenças infecciosas que fazem parte do seu diagnóstico diferencial em residentes da Região Metropolitana do Rio de Janeiro, menores de 15 anos de idade, em março de 2008.

Apesar de a técnica de sobremortalidade ser uma ferramenta útil para o aumento da sensibilidade do sistema de vigilância quando os recursos são limitados, é necessário que haja confirmação diagnóstica para afirmar que houve excesso de mortalidade por dengue.

Uma investigação, com recodificação das causas de morte nos prontuários, boletins de emergência ou qualquer outro tipo de registro do atendimento do caso das crianças que evoluíram para o óbito pelos agravos estudados em março de 2008, poderia ser realizada para identificar se a causa básica do óbito declarada era dengue.

Independente da confirmação de que houve excesso de óbitos nesse período e faixa etária, há evidências da importância de os pediatras estarem atentos aos sinais e sintomas do dengue durante a consulta médica, visando a um diagnóstico e tratamento adequados, especialmente nos períodos de maior incidência da doença.

O método de sobremortalidade é uma estratégia de monitorização ${ }^{34}$ que pode ser utilizada em tempo real, durante uma epidemia, se os dados de mortalidade estiverem disponíveis de forma oportuna ou após o final desses eventos 35 . Essa técnica, que permite a identificação precoce de alterações das condições de saúde da população pelas autoridades sanitárias 34 , possibilitando, dessa forma, que medidas de saúde pública possam ser reforçadas quando ela é mais necessária (i.e. em períodos epidêmicos), pode ser implementada em diversos softwares estatísticos. Em particular, neste projeto, foi utilizado o software livre R, em que uma rotina computacional foi desenvolvida para o processo de estimação dos parâmetros do modelo proposto. Destaca-se que pacotes estatísticos que facilitam a aplicação de forma mais automatizada estão em desenvolvimento. 


\section{Resumen}

El objetivo de este estudio es investigar el exceso de mortalidad durante la epidemia de dengue de 2007 y 2008 en la región metropolitana de Río de Janeiro, Brasil. Con el fin de predecir el número de muertes durante estos años de epidemia, un modelo de Poisson dinámico fue ajustado a los datos de mortalidad por dengue y enfermedades que son parte del diagnóstico diferencial. La inferencia se realizó bajo el paradigma bayesiano. Hubo un exceso de mortalidad en niños menores de 15 años de edad en marzo de 2008. Además, en marzo y abril de 2008, se produjo el mayor número de casos de dengue en el estado de Río de Janeiro. Considerando que el aumento de la mortalidad debe ser precedido por un aumento de la morbilidad, se puede plantear la hipótesis de que hubo un exceso de mortalidad por dengue en niños durante la epidemia de dengue que se produjo en el área metropolitana de Río de Janeiro en marzo de 2008.

Dengue; Enfermedades Transmisibles; Mortalidad; Vigilancia Epidemiológica

\section{Colaboradores}

T. A. Malhão participou da concepção do projeto, da análise e interpretação dos dados e da redação do artigo. C. M. Casquilho Resende e D. Gamerman contribuíram com a seleção do método estatístico, análise e interpretação dos dados e revisão crítica relevante do conteúdo intelectual. R. A. Medronho coordenou o projeto, participando da sua concepção, análise e interpretação dos dados e revisão crítica relevante do conteúdo intelectual.

\section{Agradecimentos}

Agradecemos à Ângela Cascão pela disponibilização do banco de dados do Sistema de Informações sobre Mortalidade, à Cláudia Medina Coeli, à Rejane Sobrino Pinheiro e aos revisores pelas ideias e sugestões, ao CNPq pelo apoio à pesquisa e à FAPERJ (processo no E-26/111.551/2008) pelo financiamento.

\section{Referências}

1. Toledo ALA, Escosteguy CC, Medronho RA, Andrade FC. Confiabilidade do diagnóstico final de dengue na epidemia 2001-2002 no Município do Rio de Janeiro, Brasil. Cad Saúde Pública 2006; 22:933-40.

2. Souza ICA, Vianna RPT, Moraes RM. Modelagem da incidência do dengue na Paraíba, Brasil, por modelos de defasagem distribuída. Cad Saúde Pública 2007; 23:2623-30.

3. Duarte HHP, França EB. Qualidade dos dados da vigilância epidemiológica da dengue em Belo Horizonte, MG. Rev Saúde Pública 2006; 40:134-42.

4. Dornas JE. Sobremortalidade durante a epidemia de dengue na cidade do Rio de Janeiro [Dissertação de Mestrado]. Rio de Janeiro: Instituto de Medicina Social, Universidade do Estado do Rio de Janeiro; 2008.

5. Oliveira FJ. A forma grave de dengue no Município do Rio de Janeiro: análise dos dados registrados nos sistemas de informação do SUS entre 2001 e 2003 [Dissertação de Mestrado]. Rio de Janeiro: Escola Nacional de Saúde Pública, Fundação Oswaldo Cruz; 2006.
6. Moraes GH, Duarte EC. Análise da concordância dos dados de mortalidade por dengue em dois sistemas nacionais de informação em saúde, Brasil, 2000-2005. Cad Saúde Pública 2009; 25:2354-64.

7. Souza AI, Nogueira JMR, Pereira MM. Anticorpos anti-leptospira em pacientes de Mato Grosso do Sul com suspeita clínica de dengue ou hepatite viral. Rev Soc Bras Med Trop 2007; 40:431-5.

8. Bandyopadhyay S, Lum LCS, Kroeger A. Classifying dengue: a review of the difficulties in using the WHO case classification for dengue haemorrhagic fever. Trop Med Int Health 2006; 11:1238-55.

9. Aguirre A, Gonzalez E. Forecast of acute respiratory infections: expected nonepidemic mobidity in Cuba. Mem Inst Oswaldo Cruz 1992; 87:433-6.

10. Simonsen L, Clarke MJ, Stroup DF, Williamson GD, Arden NH, Cox NJ. A method for timely assessment of influenza-associated mortality in the United States. Epidemiology 1997; 8:390-5.

11. Penna MLF. Rede neural artificial para detecção de sobremortalidade atribuível à cólera no Ceará. Rev Saúde Pública 2004; 38:351-7. 
12. Governo do Estado do Rio de Janeiro. Lei complementar no 105, de 4 de julho de 2002. Diário Oficial do Estado do Rio de Janeiro 2002; 5 jul.

13. Lago LC. Como anda Rio de Janeiro. Rio de Janeiro: Letra Capital/Observatório das Metrópoles; 2008. (Conjuntura Urbana, 9).

14. Secretaria de Vigilância em Saúde, Ministério da Saúde. dengue: diagnóstico e manejo clínico. 3a Ed. Brasília: Ministério da Saúde; 2007.

15. Organização Mundial da Saúde. Classificação estatística internacional de doenças, lesões e causas de óbito - 9a conferência de revisão. São Paulo: Centro Brasileiro de Classificação de Doenças em Português; 1985.

16. Organização Mundial da Saúde. Classificação estatística internacional de doenças e problemas relacionados à saúde - 10a revisão. São Paulo: Centro Colaborador da OMS para a Classificação de Doenças em Português; 1995.

17. World Health Organization. Translator: ninth and tenth revisions. User's guide to electronic tables. http://whqlibdoc.who.int/icd/hq/1996/10_to_9. pdf (acessado em 08/Mar/2009).

18. Buchalla CM, Laurenti R, Ribeiro AF, Nitrini DR, Guarnieri CE, Carnaúba EL, et al. Avaliação do uso da Classificação Internacional de Doenças para codificar a síndrome da imunodeficiência adquirida. Rev Saúde Pública 1996; 30:479-82.

19. Souza MVP. Uma abordagem bayesiana para o cálculo dos custos operacionais eficientes das distribuidoras de energia elétrica [Tese de Doutorado]. Rio de Janeiro: Departamento de Engenharia Elétrica, Pontifícia Universidade Católica do Rio de Janeiro; 2008.

20. Franco GC, Gamerman D, Santos TR. Modelos de espaço de estados: abordagens clássica e bayesiana. São Paulo: Associação Brasileira de Estatística; 2009.

21. Harvey AC, Fernandes C. Time series models for count or qualitative observations. J Bus Econ Stat 1989; 7:407-17.

22. Santos TR. Inferência sobre os hiperparâmetros em modelos estruturais sob as perspectivas clássica e bayesiana [Dissertação de Mestrado]. Belo Horizonte: Departamento de Estatística, Universidade Federal de Minas Gerais; 2009.

23. Gamerman D, Lopes HF. Markov Chain Monte Carlo: stochastic simulation for bayesian inference. New York: Chapman \& Hall/CRC Texts in Statistical Science; 2006.

24. Glezen PW. Serious morbidity and mortality associated with influenza epidemics. Epidemiol Rev 1982; 4:25-44.
25. Sindo N, Li M, Ohkusa Y, Taniguchi K. A newly developed Japanese pneumonia and influenza mortality model and statistical analysis of excess mortality by stochastic frontier estimation. Osaka: Institute of Social and Economic Research, Osaka University; 2000.

26. Imaz MS, Eimann M, Poyard E, Savy V. Exceso de mortalidad asociada a influenza en Argentina: 1992 2002. Rev Chil Infectol 2006; 23:297-306.

27. Machado SWS. Gabinete de crise: gerenciamento de epidemia no Rio de Janeiro. In: V Seminário Internacional de Defesa Civil. http:/ /www.defesacivil.uff.br/ defencil_5/Artigo_Anais_Eletronicos_Defencil_17. pdf (acessado em 20/Jan/2010).

28. Rodrigues MBP, Freire HBM, Corrêa PRL, Mendonça ML, Silva MRI, França EB. É possível identificar a dengue em crianças a partir do critério de caso suspeito preconizado pelo Ministério da Saúde? J Pediatr (Rio J.) 2005; 81:209-15.

29. Nunes-Araújo FR, Ferreira MS, Nishioka A. Dengue fever in Brazilian adults and children: assessment of clinical findings and their validity for diagnosis. Ann Trop Med Parasitol 2003; 97:415-9.

30. Figueiredo LT, Cavalcante SM, Simões MC. Encuesta serológica sobre el dengue entre escolares de Rio de Janeiro, Brasil, 1986 y 1987. Bol Oficina Sanit Panam $1991 ; 111: 525-33$.

31. Kalayanarooj S, Vaughn DW, Nimmannitya S, Green S, Suntayakorn S, Kunentrasai N, et al. Early clinical and laboratory indicators of acute dengue illness. J Infect Dis 1997; 176:313-21.

32. Teeraratkul A, Limpakarnjanara K, Nisalak A, Nimmannitya S. Predictive value of clinical and laboratory findings for early diagnosis of dengue and dengue hemorrhagic fever. Southeast Asian J Trop Med Public Health 1990; 21:696-7.

33. Graham RR, Juffrie M, Tan R, Hayes CG, Laksono I, Ma'roef $\mathrm{C}$, et al. A prospective seroepidemiologic study on dengue in children four to nine years of age in Yogyakarta, Indonesia I. studies in 1995-1996. Am J Trop Med Hyg 1999; 61:412-9.

34. Gerolomo M, Penna MLF. Sobremortalidade por diarreia simultânea à cólera na Região Nordeste do Brasil. Rev Saúde Pública 2004; 38:517-22.

35. Ramchurn SK, Goorah SS, Makhan M, Moheeput $\mathrm{K}$. Excess mortality as an epidemic intelligence tool in chikungunya mapping. Euro Surveill 2008; 13:pii:8039.

Recebido em 17/Mai/2012

Versão final reapresentada em 06/Fev/2013 Aprovado em 08/Mai/2013 